\title{
ARE T TAURI STARS GAMMA-RAY EMITTERS?
}

\author{
María Victoria del Valle ${ }^{1,2}$, Gustavo E. Romero ${ }^{1,2}$, Pedro Luis Luque-Escamilla ${ }^{3}$, \\ Josep Martí ${ }^{4}$, AND JuAN RAMÓN SÁNChEZ-SutiL ${ }^{4}$ \\ ${ }^{1}$ Instituto Argentino de Radioastronomía (IAR), CCT La Plata (CONICET), C.C.5, (1894) Villa Elisa, Buenos Aires, Argentina \\ ${ }^{2}$ Facultad de Ciencias Astronómicas y Geofísicas, Universidad Nacional de La Plata, Paseo del Bosque s/n, 1900 La Plata, Argentina \\ ${ }^{3}$ Departamento de Ingeniería Mecánica y Minera, Escuela Politécnica Superior de Jaén, Universidad de Jaén, \\ Campus Las Lagunillas s/n, A3, 23071 Jaén, Spain \\ ${ }^{4}$ Departamento de Física, Escuela Politécnica Superior de Jaén, Universidad de Jaén, \\ Campus Las Lagunillas s/n, A3, 23071 Jaén, Spain \\ Received 2011 March 22; accepted 2011 June 28; published 2011 August 17
}

\begin{abstract}
T Tauri stars are young, low-mass, pre-main-sequence stars surrounded by an accretion disk. These objects present strong magnetic activity and powerful magnetic reconnection events. Strong shocks are likely associated with fast reconnection in the stellar magnetosphere. Such shocks can accelerate particles up to relativistic energies. We aim at developing a simple model to calculate the radiation produced by non-thermal relativistic particles in the environment of T Tauri stars. We want to establish whether this emission is detectable at high energies with the available or forthcoming $\gamma$-ray telescopes. We assume that particles (protons and electrons) pre-accelerated in reconnection events are accelerated at shocks through the Fermi mechanism and we study the high-energy emission produced by the dominant radiative processes. We calculate the spectral energy distribution of $\mathrm{T}$ Tauri stars up to high energies and we compare the integrated flux obtained with that from a specific Fermi source, 1FGL J1625.8-2429c, that we tentatively associate with this kind of young stellar object. We suggest that under reasonable general conditions nearby $\mathrm{T}$ Tauri stars might be detected at high energies and be responsible for some unidentified Fermi sources on the Galactic plane.
\end{abstract}

Key words: gamma rays: stars - radiation mechanisms: non-thermal - stars: coronae - stars: flare - stars: protostars

\section{INTRODUCTION}

The Fermi Gamma-Ray Space Telescope was launched on 2008 June 11 and is the successor of the Compton GammaRay Observatory. It has two $\gamma$-ray instruments on board, the Large Area Telescope (LAT) and the Gamma-ray Burst Monitor (GBM). The first one, a pair-production telescope, is the successor of the Energetic Gamma-Ray Experiment Telescope (EGRET). LAT has a sensitivity $\sim 10^{-9} \mathrm{ph} \mathrm{s}^{-1} \mathrm{~cm}^{-2}$ in the energy range from $20 \mathrm{MeV}$ to over $300 \mathrm{GeV}$.

Based on the first 11 months of survey, the Fermi First Year Catalog has been published (Abdo et al. 2010). The catalog consists of 1451 sources with statistical significances of $4 \sigma$ or higher. This catalog provides potential identifications, when available. Nearly 630 Fermi sources remained unidentified (Abdo et al. 2010). A fraction of these unidentified sources lay on the Galactic plane (latitudes $\leqslant 5^{\circ}$ ). They may be pulsars, supernova remnants (SNRs), the effect of strong winds from massive stars, high-mass or low-mass X-ray binaries (HMXBs or LMXBs), or stellar clusters, among other possibilities (e.g., Romero et al. 1999).

Munar-Adrover et al. (2011) have crossed the Fermi First Year Catalog with catalogs of known young stellar objects (YSOs), in order to identify those protostars that might emit $\gamma$-rays. They conclude that $72 \%$ of the candidates obtained by spatial correlation should be $\gamma$-ray sources with a confidence above $5 \sigma$. Massive YSOs have already been claimed to be $\gamma$-ray sources (e.g., Araudo et al. 2007; Bosch-Ramon et al. 2010). However, such a claim has not been done yet for low-mass protostars.

In this paper, we shall argue that these stars, in particular $\mathrm{T}$ Tauri stars, can be faint but sometimes detectable $\gamma$-ray sources. We will focus our attention on the physical processes that can generate $\gamma$-ray emission in T Tauri magnetospheres.
Specifically, we shall discuss whether these protostars can be responsible for sources like 1FGL J1625.8-2429c. In the next section, we present a brief introduction to $\mathrm{T}$ Tauri stars and we describe the general physical scenario. In Section 3, we provide the details of our model. In Section 4, we present our calculations applied to a specific group of T Tauri stars and the main results. In Section 5, we discuss these results and, finally, we close with some brief conclusions.

\section{SCENARIO}

T Tauri stars are low-mass stars $\left(M<3 M_{\odot}\right)$ in their early stages of evolution. Classical $\mathrm{T}$ Tauri stars have typical K-M spectral types with $T_{\text {eff }} \sim 3000-5000 \mathrm{~K}$ (Montmerle \& André 1989). Values of the stellar radius are $\sim 2-3 R_{\odot}$. As progenitors of solar-like stars, they are the subject of intense study. They are usually optically visible and the youngest objects drive bipolar outflows. The protostars are born in the collapse of molecular clouds. The cloud material has high angular momentum and therefore a circumstellar accretion disk is formed (e.g., Beckwith et al. 1990). These accretion disks are truncated in the vicinity of a co-rotation radius where the magnetic pressure equals the gas pressure in the disk (see Figure 1). Infrared observations allow inner radii of 0.07-0.54 AU (Muzerolle et al. 2003) to be inferred, consistent with the co-rotation radius. UV emission has been used to estimate the mass accretion rate, yielding typical values of $\sim 10^{-8} M_{\odot}$ year $^{-1}$ (Gomez de Castro \& Lamzin 1999; JohnsKrull et al. 2000). The infall velocity can almost reach the freefall speed. The existence of a large amount of dust in a flattened disk is confirmed by intense emission from the micrometer to the millimeter bands. The disks can now be directly imaged (e.g., Dutrey et al. 1994). In addition, these objects drive strong 


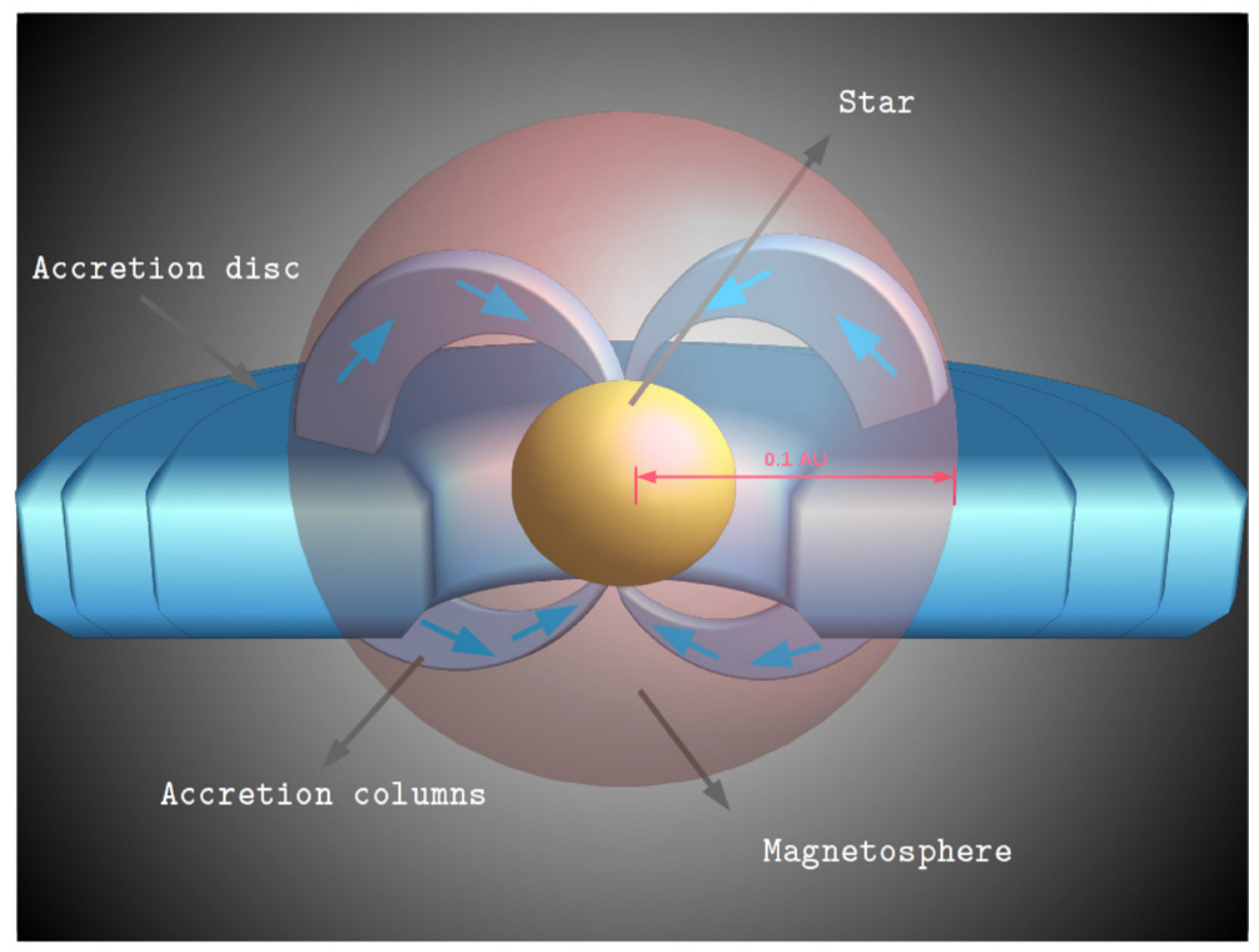

Figure 1. Sketch of a T Tauri star adapted from Feigelson \& Montmerle (1999).

winds with mass-loss rate $\dot{M} \sim 10^{-8} M_{\odot}$ year $^{-1}$ and velocity $v_{\mathrm{w}} \sim 200 \mathrm{~km} \mathrm{~s}^{-1}$ (Feigelson \& Montmerle 1999).

$\mathrm{X}$-ray studies indicate particle number densities of the accreting plasma of about $10^{12} \mathrm{~cm}^{-3}$ (Günther et al. 2007). Variable thermal X-ray emission is also detected from $\mathrm{T}$ Tauri stars in the keV band. Luminosities are found to be in the range $\sim 10^{31}$ $10^{33} \mathrm{erg} \mathrm{s}^{-1}$. This emission comes from a high density plasma at a typical temperature of $\sim 10^{7} \mathrm{~K}$; flares with temperatures $\sim 10^{8} \mathrm{~K}$ have been detected (Tsuboi et al. 1998). These flares have durations of $\sim 10^{3}-10^{4} \mathrm{~s}$. Such events are expected to occur in magnetic flux tubes with spatial extent of $\sim 10^{10}-10^{11} \mathrm{~cm}$ (e.g., Hayashi et al. 1996). Models for the X-ray activity based on the interaction of the stellar object and the circumstellar disk have been proposed by several authors (e.g., Hayashi et al. 1996; Birk et al. 2000).

These X-ray flares are widely considered as upscaled versions of solar flares. The rapid heating and cooling of plasma and acceleration of particles must arise from efficient MHD processes, such as solar-type magnetic reconnection events in twisted flux tubes that connect the central object and the circumstellar disk (Birk et al. 2000). Magnetic reconnection is one of the fundamental processes in astrophysical plasmas because it explains large-scale, dynamic releases of magnetic energy. It is essentially a topological reconfiguration of the magnetic field caused by a change in the connectivity of the field lines. It is this change which allows the release of stored magnetic energy, which in many situations is the dominant source of free energy in a plasma. Several works have been done on particle acceleration through magnetic reconnection (e.g., Schopper et al. 1998; Zenitani \& Hoshino 2001; de Gouveia Dal Pino et al. 2010). Strong shocks resulting from supersonic plasma ejections are the likely outcome of massive reconnection in T Tauri magnetospheres. Such shocks can in principle accelerate particles up to relativistic energies through a Fermi mechanism.

The expected values of magnetic field in $\mathrm{T}$ Tauri stars are $\sim 1 \mathrm{kG}$ (e.g., Johns-Krull 2007) and the field structure is complex and multipolar, as in the Sun. For simple flare models, quantitative properties of large-scale magnetic field structures can be inferred (e.g., Montmerle et al. 1983; Walter \& Kuhi 1984). Assuming equipartition conditions, $B_{\text {eq }}^{2} / 8 \pi=2 n_{\mathrm{e}} k T$, the magnetic field strength in the magnetosphere is approximately $10^{2} \mathrm{G}$ (Feigelson \& Montmerle 1999).

\section{MODEL}

\subsection{Particle Acceleration}

Diffusive shock acceleration does not work for slow-mode shocks, consequently it is thought that diffusive shock acceleration is not important for magnetic reconnection that involves slow-mode shocks (e.g., Priest \& Forbes 2000). Under some conditions, however, any obstacle which obstructs the outflow will create a fast-mode shock (Tsuneta \& Naito 1998). In a T Tauri magnetosphere, the obstacles might be clumps from the strong stellar wind; considerable observational evidence supports the idea that the wind structure is clumpy (e.g., Owocki $\&$ Cohen 2006). Moreover, shock acceleration is now applied to the outflow regions of coronal magnetic reconnection sites, where a first-order Fermi mechanism at the standing fast shock is a leading candidate (Aschwanden 2008). The shocks are expected to accelerate charged particles up to high energies by a Fermi-like diffusive process (e.g., Drury 1983).

Additionally, recent extensive three-dimensional numerical simulations performed by Kowal et al. (2011) show that within contracting magnetic islands or current sheets, charged particles are accelerated by a first-order Fermi process while outside the current sheets and islands the particles experience mostly drift acceleration due to magnetic field gradients. These results are supported by observations of solar flares that suggest that magnetic reconnection should be first slow in order to ensure the accumulation of magnetic flux and then suddenly become fast to allow a rapid energy release (e.g., Lazarian \& Vishniac 1999). Particles scattered by turbulence between converging magnetic 


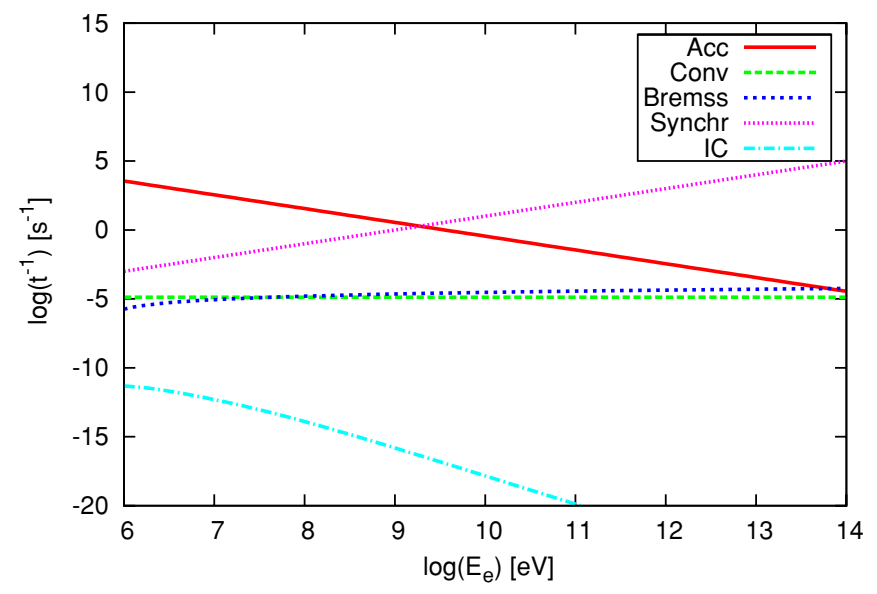

Figure 2. Acceleration and cooling rates for electrons. The identification of the different curves is given in the box in the upper right corner.

mirrors formed by oppositely directed magnetic fluxes moving toward each other at the fast reconnection speed can undergo diffusive acceleration without the requirement of strong shock formation (Kowal et al. 2011).

Independently of the details of the acceleration mechanism, we assume that a population of non-thermal relativistic particles, electrons and protons, is injected into the magnetosphere of the T Tauri star. These particles will interact with the largescale magnetic fields, with the existing radiation fields, and with the magnetospheric plasma, producing non-thermal electromagnetic radiation. The size of the acceleration region is the spatial scale where reconnection takes place, i.e., the flux tube length.

The power available in the magnetized system is

$$
L=\frac{B^{2}}{8 \pi} A v_{\mathrm{A}},
$$

where $A$ is the area of the flux tube, of length $l=10^{11} \mathrm{~cm}$ and aspect ratio $\sim 0.1 l$ (Feigelson \& Montmerle 1999), and $v_{\mathrm{A}}$ is the Alfvén speed, $v_{\mathrm{A}}=\sqrt{B^{2} /\left(4 \pi m_{p} n\right)}$, with $n$ the particle density and $m_{p}$ the proton mass. For $B=2 \times 10^{2} \mathrm{G}$, we get $L \sim 10^{34} \mathrm{erg} \mathrm{s}^{-1}$. We assume that $10 \%$ of this power is released in the reconnection processes. In turn, a fraction $q_{\text {rel }} \sim 10 \%$ of this power goes to relativistic particles. These values are in accordance with inferred values for the Sun, and can be considered even conservative. For instance, in large solar flares the accelerated particles contain up to $10 \%-50 \%$ or more of the total energy released, whereas in gradual events $\sim 10 \%$ of the total power goes to the accelerated particles (see Lin 2008, and references therein).

The efficiency of non-thermal acceleration in the magnetized plasma is

$$
\eta \sim 10^{-1} \frac{r_{\mathrm{g}} c}{D}\left(\frac{v_{\mathrm{rec}}}{c}\right)^{2},
$$

mimicking the efficiency for standard first-order Fermi acceleration theory behind shocks ${ }^{5}$ (Drury 1983; Vila \& Aharonian 2009); here $D$ is the particle diffusion coefficient, $r_{\mathrm{g}}$ is the particle gyroradius, and $v_{\text {rec }}$ is the reconnection speed. If $D$ is in the

\footnotetext{
5 This assumption holds only if there is a first-order Fermi mechanism occurring within the reconnection zone caused by two converging magnetic fluxes of opposite polarity which move to each other with $v_{\text {rec }}$. This mechanism of first-order Fermi acceleration occurring within a reconnection site was first proposed and described analytically in de Gouveia Dal Pino \& Lazarian (2005) and successfully tested through three-dimensional MHD simulations by Kowal et al. (2011).
}

Table 1

\begin{tabular}{|c|c|}
\hline Parameter (units) & Values \\
\hline$l \quad$ Flux tube length $(\mathrm{cm})$ & $10^{11}$ \\
\hline$v_{\mathrm{A}} \quad$ Alfvén speed $\left(\mathrm{cm} \mathrm{s}^{-1}\right)$ & $6 \times 10^{7}$ \\
\hline$\eta \quad$ Acceleration efficiency & $10^{-6}$ \\
\hline a Hadron-to-lepton energy ratio & 100 \\
\hline$q_{\text {rel }}$ Fractional content of relativistic particles & $10^{-1}$ \\
\hline$\alpha \quad$ Particle injection index & 2.2 \\
\hline$v_{\mathrm{W}}$ Wind velocity $\left(\mathrm{cm} \mathrm{s}^{-1}\right)$ & $2 \times 10^{7}$ \\
\hline$B \quad$ Magnetic field $(\mathrm{G})$ & $2 \times 10^{2}$ \\
\hline$n \quad$ Particle density $\left(\mathrm{cm}^{-3}\right)$ & $5 \times 10^{11}$ \\
\hline
\end{tabular}

Parameters

Bohm limit $D_{\text {Bohm }}=r_{\mathrm{g}} c / 3$. The reconnection speed in violent reconnection events satisfies $v_{\text {rec }} \sim v_{\mathrm{A}}$ (Lazarian \& Vishniac 1999; Kowal et al. 2009), so we assume $v_{\text {rec }}=0.6 v_{\mathrm{A}}$ that gives an efficiency $\eta \sim 10^{-6}$. This efficiency, although not very high, will allow maximum energies well into the $\gamma$-ray domain, as we will see.

\subsection{Energy Losses}

The relativistic electrons and protons lose energy through different interactions. The maximum energy that they can reach depends on the processes of energy loss, the available space for effective acceleration, and on the acceleration rate. This rate is $t_{\mathrm{acc}}^{-1}=\eta e c B / E$ for the first-order Fermi mechanism.

The main loss mechanism for electrons in the magnetosphere of T Tauri stars is synchrotron radiation. Other relevant loss processes are relativistic Bremsstrahlung, the leptons interact with ions of material in the magnetosphere, and inverse Compton scattering (IC) - electrons scatter up the ambient photons. The main ambient photon field is the thermal $\mathrm{X}$-ray emission, which corresponds to a temperature of $\sim 10^{7} \mathrm{~K}$, and has a typical luminosity (in quiescence) of $\sim 6 \times 10^{29} \mathrm{erg} \mathrm{s}^{-1}$ according to the observed values in this paper.

Figure 2 shows the cooling rates and the acceleration rate for electrons in the case of the parameters shown in Table 1. These parameters are fixed in accordance with the typical values discussed above for T Tauri stars (see, e.g., Feigelson \& Montmerle 1999). Particles can also escape by convection from the acceleration region with a rate $t_{\text {conv }}^{-1}=v_{\mathrm{w}} / l$, where $v_{\mathrm{w}}$ is the wind velocity. The maximum energy for electrons is obtained equating the cooling rates with the acceleration rate. The result is $\sim 2 \mathrm{GeV}$. It can be seen that electrons rapidly cool by a synchrotron mechanism with timescales of less than $1 \mathrm{~s}$.

The radiative losses for protons are $p \gamma$ interactions with the $\mathrm{X}$-ray radiation field, synchrotron and $p p$ inelastic collisions. The latter losses are the most important for these particles. Figure 3 shows the cooling rates and the acceleration rate for protons. The maximum energy of these particles is much greater than the maximum energy of electrons, $\sim 10 \mathrm{TeV}$. Protons of such an energy satisfy the Hillas criterion for a field of $2 \times 10^{2} \mathrm{G}$. Acceleration timescales at the higher energies are $\sim 10^{5} \mathrm{~s}$, larger than the typical durations of flaring X-ray episodes $\left(\sim 10^{4} \mathrm{~s}\right)$. Hence, maximum energies of $\sim 10 \mathrm{TeV}$ are more realistic in a steady-state approximation to the $\gamma$-ray active period.

\subsection{Particle Distribution}

We assume an injection function $Q(E)$ that is a power law, of index 2.2, in the energy of the particles (Drury 1983)

$$
Q(E)=Q_{0} E^{-\alpha} e^{-E / E_{\max }} .
$$




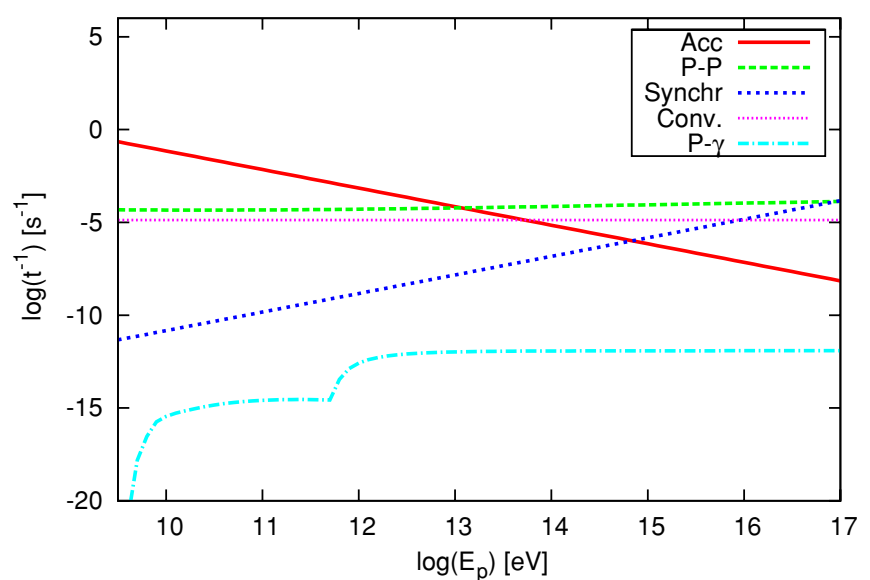

Figure 3. Acceleration and cooling rates for protons.

The normalization constant $Q_{0}$ for each kind of particle is obtained from

$$
L_{\mathrm{e}}=\frac{L_{\mathrm{rel}}}{1+a}=V \int_{E_{\min }}^{E_{\max }} Q_{0} E^{-\alpha} E d E,
$$

and

$$
L_{p}=\frac{L_{\mathrm{rel}}}{1+1 / a}=V \int_{E_{\min }}^{E_{\max }} Q_{0} E^{-\alpha} E d E .
$$

Here, $a$ is the hadron-to-lepton energy density ratio. We consider $a=100$ as observed in cosmic rays in the solar neighborhood (Ginzburg \& Syrovatskii 1964), $L_{\mathrm{rel}}$ is the power in the form of relativistic particles and $V$ is the acceleration volume, i.e., the volume of the flux tube. The steady-state particle distributions $N(E)$ are obtained solving the transport equation in the homogeneous approximation

$$
\frac{\partial}{\partial E}\left[\left.\frac{d E}{d t}\right|_{\text {loss }} N(E)\right]+\frac{N(E)}{t_{\text {esc }}}=Q(E),
$$

where $t_{\text {esc }}=t_{\text {conv }}$. This equation has an exact analytical solution (see Ginzburg \& Syrovatskii 1964):

$$
N(E)=\left|\frac{d E}{d t}\right|_{\mathrm{loss}}^{-1} \int_{E}^{E^{\max }} d E^{\prime} Q\left(E^{\prime}\right) \exp \left(-\frac{\tau\left(E, E^{\prime}\right)}{t_{\mathrm{esc}}}\right),
$$

with

$$
\tau\left(E, E^{\prime}\right)=\int_{E}^{E^{\prime}} d E^{\prime \prime}\left|\frac{d E^{\prime \prime}}{d t}\right|_{\text {loss }}^{-1} .
$$

Figures 4 and 5 show the steady-state particle distribution for protons and electrons. These distributions are valid only as long as we consider intervals much shorter than the timescale of the flare.

\subsection{Luminosity}

In order to estimate the luminosity due to electrons we computed synchrotron, relativistic Bremsstrahlung, synchrotron self-Compton (SSC) radiation, and IC up scattering of external seed photons (X-ray and IR radiation fields). We calculate for protons synchrotron and $\gamma$-ray emission from $\pi^{0}$ decay in $p p$ inelastic collisions. We consider also the synchrotron contribution from secondary pairs injected by charged pion decay (e.g., Orellana et al. 2007). The maximum particle density is $\sim 5 \times 10^{11} \mathrm{~cm}^{-3}$. It is localized in the accretion columns. We

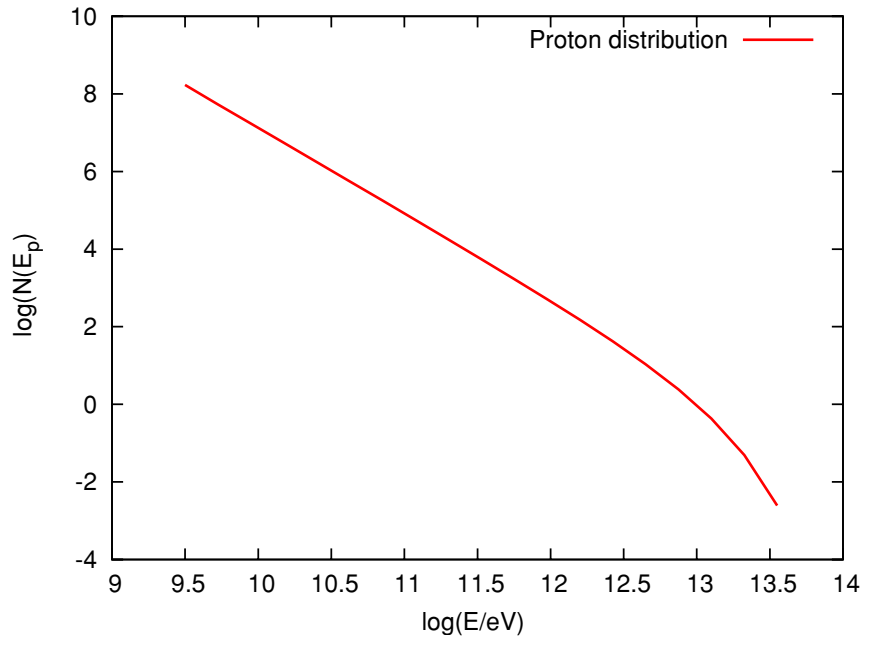

Figure 4. Steady-state particle distribution for protons.

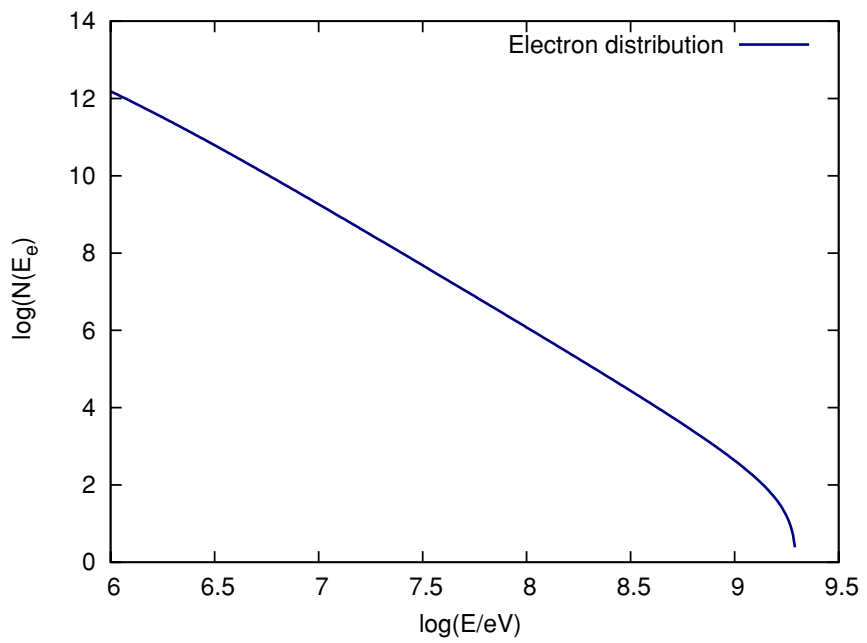

Figure 5. Steady-state particle distribution for electrons.

consider a cylindrical portion of the accretion column of radius $r_{\mathrm{AC}} \sim 10^{10} \mathrm{~cm}$ and height $\sim 0.1 r_{\mathrm{AC}}$ (e.g., Orlando et al. 2010). This volume is where most of the relativistic interactions with matter take place. ${ }^{6}$ For more details on high-energy processes, see Vila \& Aharonian (2009) and Vila (2010).

\subsubsection{Internal Absorption}

Attenuation of the radiation produced by photon annihilation is expected in T Tauri stars. The opacity produced by a photon field with density $n_{\mathrm{ph}}(\epsilon)$ and photon energy $\epsilon$ is

$$
\begin{aligned}
\tau\left(E_{\gamma}\right)= & \frac{1}{2} \int_{l} \int_{\epsilon_{\mathrm{th}}}^{\epsilon_{\max }} \int_{-1}^{u_{\max }}(1-u) \sigma_{\gamma \gamma}\left(E_{\gamma}, \epsilon, u\right) n_{\mathrm{ph}}(\epsilon) . \\
& \times d u d \epsilon d l
\end{aligned}
$$

Here, $u=\cos \vartheta, \vartheta$ is the angle between the momenta of the colliding photons, $l$ is the photon path, and $\sigma_{\gamma \gamma}\left(E_{\gamma}, \epsilon, u\right)$ is the cross section for photon annihilation (Gould \& Schréreder 1967).

The absorbing photon fields are those generated within the system and the strong blackbody radiation field from the

\footnotetext{
6 The accretion disk is truncated at the edge of the magnetosphere. The exterior disk material has no major impact in the calculations since the interacting matter is in the accreting columns that penetrate the magnetosphere.
} 


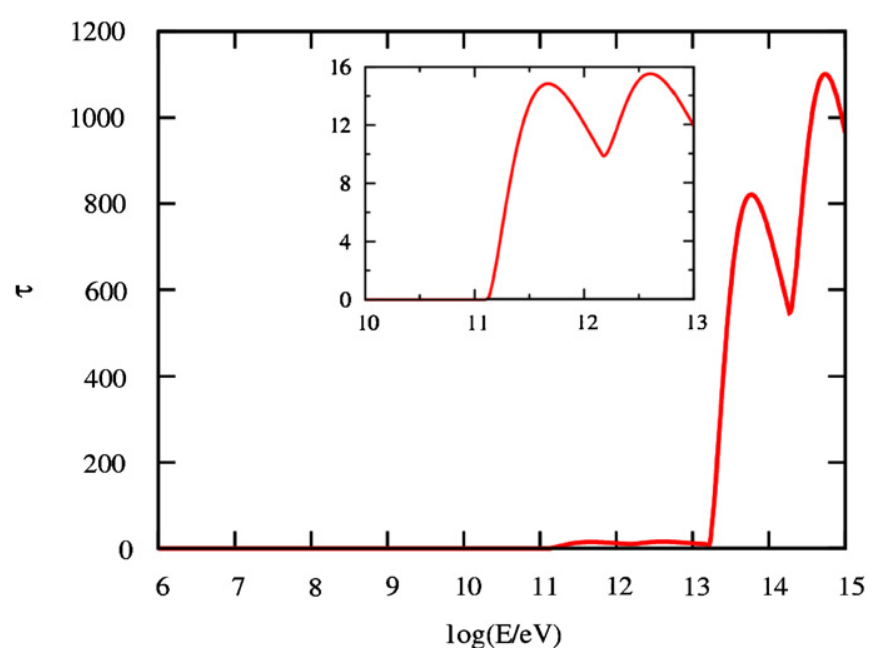

Figure 6. Opacity curve as a function of energy $E$.

Table 2

Fermi Integrated Fluxes and Calculated Fluxes

\begin{tabular}{lcc}
\hline \hline Energy Range & Flux \pm Error $\left(\mathrm{cm}^{-2} \mathrm{~s}^{-1}\right)$ & Computed Flux \\
\hline $100 \mathrm{MeV}-300 \mathrm{MeV}$ & $1.9 \times 10^{-7} \pm 1.0 \times 10^{-8}$ & $6.30 \times 10^{-8}$ \\
$300 \mathrm{MeV}-1 \mathrm{GeV}$ & $2.2 \times 10^{-8} \pm 0.6 \times 10^{-8}$ & $1.89 \times 10^{-8}$ \\
$1 \mathrm{GeV}-3 \mathrm{GeV}$ & $4 \times 10^{-9} \pm 0.7 \times 10^{-9}$ & $4.82 \times 10^{-9}$ \\
$3 \mathrm{GeV}-10 \mathrm{GeV}$ & $8.5 \times 10^{-10} \pm 2.2 \times 10^{-10}$ & $1.21 \times 10^{-9}$ \\
$10 \mathrm{GeV}-100 \mathrm{GeV}$ & $2.1 \times 10^{-10} \pm 1.0 \times 10^{-11}$ & $3.76 \times 10^{-10}$
\end{tabular}

surroundings: the disk (IR), the star, and the X-rays from the accreting plasma. To estimate the disk infrared photon field, we adopt an internal radius for the disk of $R_{\mathrm{D}} \sim 120 \mathrm{AU}$ (see Figure 1), and a temperature of $T \sim 30 \mathrm{~K}$ (Dutrey et al. 1994). The X-ray temperature is taken to be $T \sim 10^{7} \mathrm{~K}$ (e.g., Feigelson \& Montmerle 1999). Regarding the star, we consider a temperature of $T_{\star} \sim 4 \times 10^{3} \mathrm{~K}$, and a radius $R_{\star} \sim 2 R_{\odot}$. The opacity $\tau$ (attenuation $e^{-\tau}$ ) as a function of $E_{\gamma}$ is shown in Figure 6. The absorption is almost complete above $100 \mathrm{GeV}$.

\section{APPLICATION TO POSSIBLE $\gamma$-RAY EMITTING T TAURI STARS}

After performing several catalog cross-correlations, a new possible association with nearby $\mathrm{T}$ Tauri stars inside the wellknown $\rho$ Ophiuchi star-forming region clearly emerges for the Fermi source 1FGL J1625.8-2429c. Indeed, inside the 95\% confidence error ellipse of this Fermi source we find four T Tauri stars: 2MASS J16260160-2429449 (Casanova et al. 1995), 2MASS J16253958-2426349 (Wilking et al. 1989), JCMTSF J162556.8-243014 (Di Francesco et al. 2008), and 2MASS J16255752-2430317 (Grasdalen et al. 1973). Their observational properties are summarized in Table 3. In Figures $7-9$ we show this field $(l=353.0, b=17.0)$ as ob- served in the infrared, X-ray, and radio wavelengths. These images have been produced using public data retrieved (and calibrated when necessary) from the Spitzer-GLIMPSE, Chandra, and NRAO Very Large Array (VLA) archives, respectively. As discussed below, we tentatively suggest that this Fermi source might be the result of the emission of at least these four T Tauri stars that lay inside the location error box of 1FGL J1625.82429c.

In order to estimate the a priori probability of a pure chance association, we have implemented Monte Carlo simulations of computer-generated Fermi sources following the approach developed by Romero et al. (1999) for unidentified EGRET sources. After $10^{4}$ simulations of artificial Fermi populations, we find 47 coincidences at $1^{\circ}$ binning and 4 at $2^{\circ}$ binning, indicating a probability of chance association of $\sim 10^{-3}$. These results do not change with larger samples (we run up to $10^{6}$ simulations).

Let $F\left(E_{1}, E_{2}\right) \pm \triangle\left[F\left(E_{1}, E_{2}\right)\right]$ be the integrated Fermi flux in the energy range $\left[E_{1}, E_{2}\right]$ and its error. To reproduce the observed fluxes, we consider in first approximation that the four $\mathrm{T}$ Tauri stars emit the same $\gamma$-ray luminosity. Then, we can compute the integrated flux from:

$$
F=4 \int_{E_{1}}^{E_{2}} \frac{L_{\gamma}(E)}{4 \pi d^{2} E^{2}} d E .
$$

Here, $L_{\gamma}(E)$ is the total $\gamma$-ray luminosity produced by an individual T Tauri star according to our model $(E>20 \mathrm{MeV})$ and $d$ is the distance to the $\rho$ Ophiuchi cloud, $\sim 120 \mathrm{pc}$ (Loinard et al. 2008). The integrated Fermi fluxes (Abdo et al. 2010) and the calculated fluxes in five energy bands are shown in Table 2. Figure 10 shows the luminosity obtained with the model and the upper bound given by Chandra and Fermi data. Radio data from VLA are also shown, but this radiation is surely a combination of non-thermal and thermal emission and hence must be considered just as an upper value to constrain the model.

\section{DISCUSSION}

We see that for a reasonable set of physical parameters of $\mathrm{T}$ Tauri stars, a relatively weak $\gamma$-ray source can be produced. The intrinsic luminosity is not high $\left(L_{\gamma} \sim 10^{31} \mathrm{erg} \mathrm{s}^{-1}\right.$ at $\sim 1 \mathrm{GeV}$ ), but since the stars are very nearby the flux is significant. If such an association is confirmed, the $\mathrm{T}$ Tauri stars in $\rho$ Ophiuchi would be the nearest $\gamma$-ray sources to the solar system detected so far. A caveat is in order, however, considering the potential effects of the galactic cosmic rays with the molecular material in $\rho$ Ophiuchi. This emission has been subtracted from the Fermi $\gamma$-ray data (Abdo et al. 2010), but there could be more matter than detected so far.

In the case that matter is underestimated in $\rho$ Ophiuchi, we calculate the required density excess to explain the origin of the high-energy emission from the source 1FGL J1625.8-2429c.

Table 3

Properties of T Tauri Stars Proposed as 1FGL J1625.8-2429c Counterpart

\begin{tabular}{lccccc}
\hline \hline Star No. & Name & Mag. & $\begin{array}{c}\text { X-Ray Counterpart } \\
\text { CXO }\end{array}$ & $\begin{array}{c}0.5-7 \text { keV Flux } \\
\left(\mathrm{erg} \mathrm{s}^{-1} \mathrm{~cm}^{-2}\right)\end{array}$ & $\begin{array}{c}6 \mathrm{~cm} \mathrm{Flux} \mathrm{Density} \\
(\mathrm{mJy})\end{array}$ \\
\hline 1 & 2MASS J16260160-2429449 & $R=17.2$ & $\mathrm{~J} 162601.6-242945$ & $2.30 \times 10^{-13}$ & $4.52 \times 10^{-13}$ \\
2 & 2MASS J16255752-2430317 & $V=16.4$ & $\mathrm{~J} 162557.5-243031$ & $\ldots$ & $\ldots$ \\
3 & JCMTSF J162556.8-243014/ & $R=16.8$ & $\mathrm{~J} 162556.0-243014$ & $4.95 \times 10^{-13}$ & $0.30 \pm 0.03$ \\
& 2MASS J16255609-2430148 & & & & \\
4 & 2MASS J16253958-2426349 & $V=19.1$ & $\mathrm{~J} 162539.5-242634$ & $4.90 \times 10^{-14}$ & $\ldots$ \\
\hline
\end{tabular}




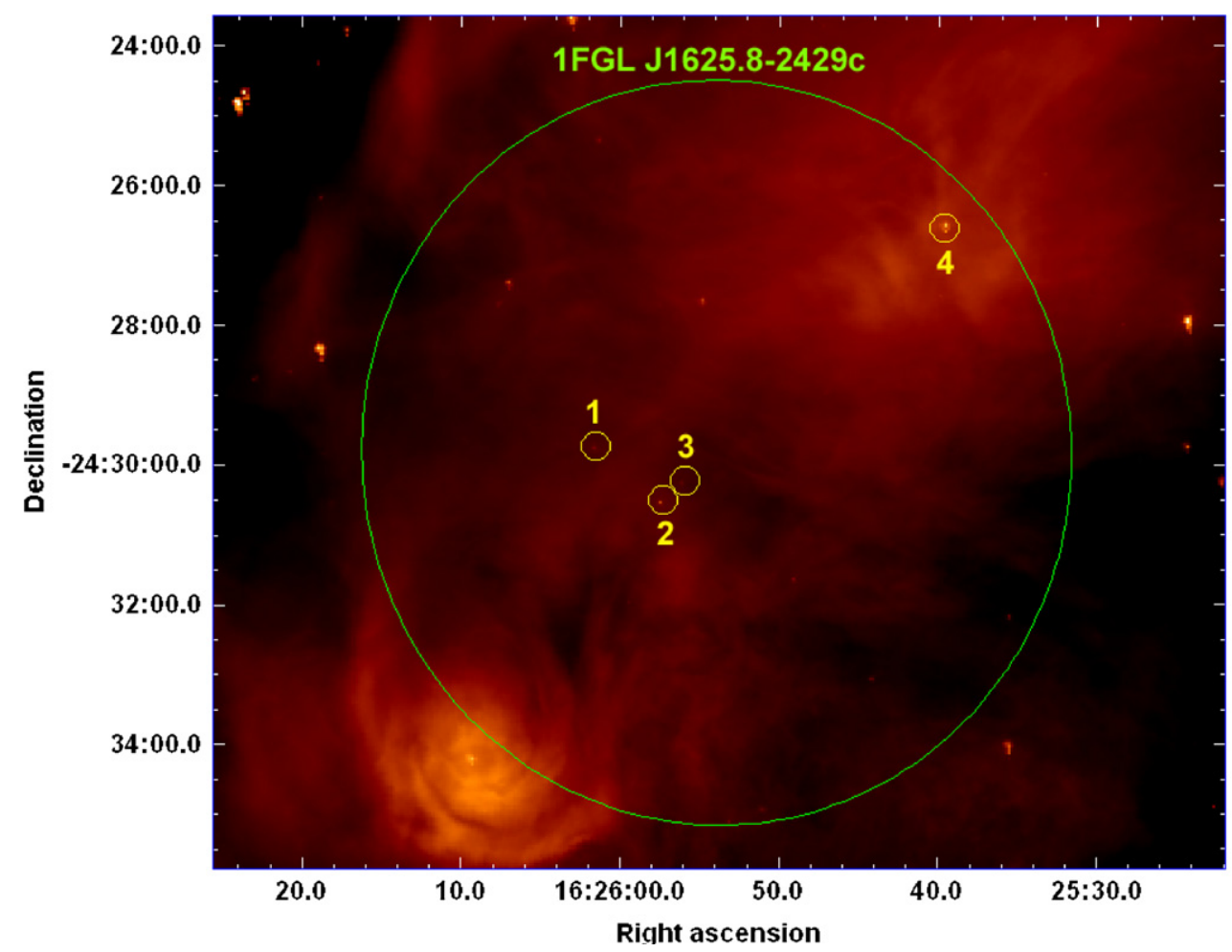

Figure 7. GLIMPSE infrared image in the $8.0 \mu \mathrm{m}$ band showing the contents of the 1FGL J1625.8-2429c error circle toward the $\rho$ Ophiuchi cloud. Several T Tauri stars are consistent with the Fermi $\gamma$-ray source position. They are labeled from 1 to 4 in decreasing order or right ascension. Axis coordinates are of equatorial J2000.0-type.

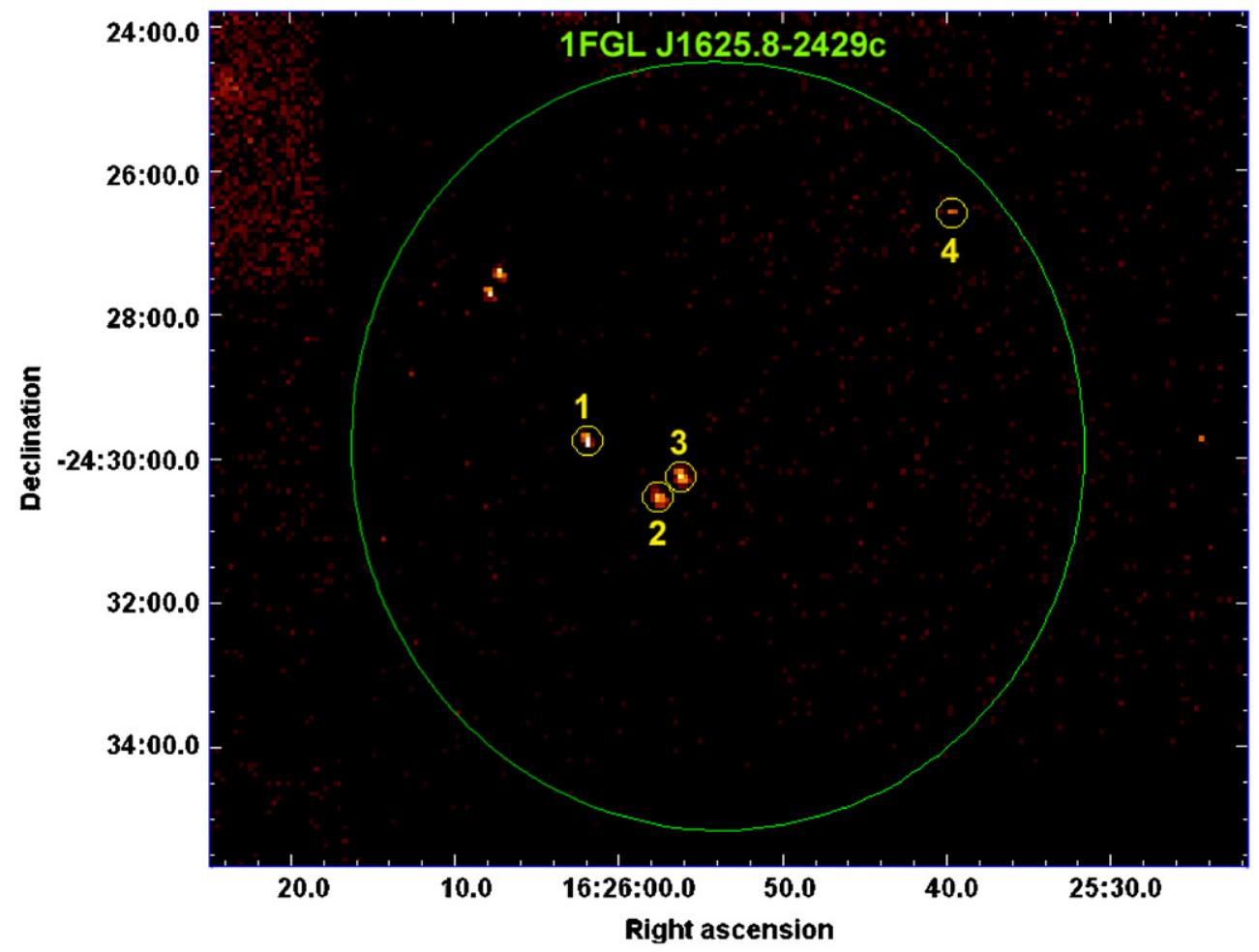

Figure 8. Composite X-ray image of the 1FGL J1625.8-2429c error circle obtained with the Chandra ACIS camera in the energy range 0.1-10 keV (data set identifier: ADS/Sa.CXO\#obs/00618). Numbers indicate the T Tauri stars consistent with this Fermi source in decreasing order of right ascension. All of these stars are X-ray emitters.

With this aim we assume that the whole $\gamma$-ray source is the result of passive "illumination" of cloud material by cosmic rays (e.g., Aharonian \& Atoyan 1996). We adopt a standard cosmic-ray spectrum in $\rho$ Ophiuchi. Proton-proton inelastic collisions with the ambient medium are the main radiative process contributing to the high-energy electromagnetic spectrum. The cosmic-ray proton spectrum has a power law of index $\alpha=2.7$ and the enhancement parameter is taken to be 1 (i.e., absence of local 


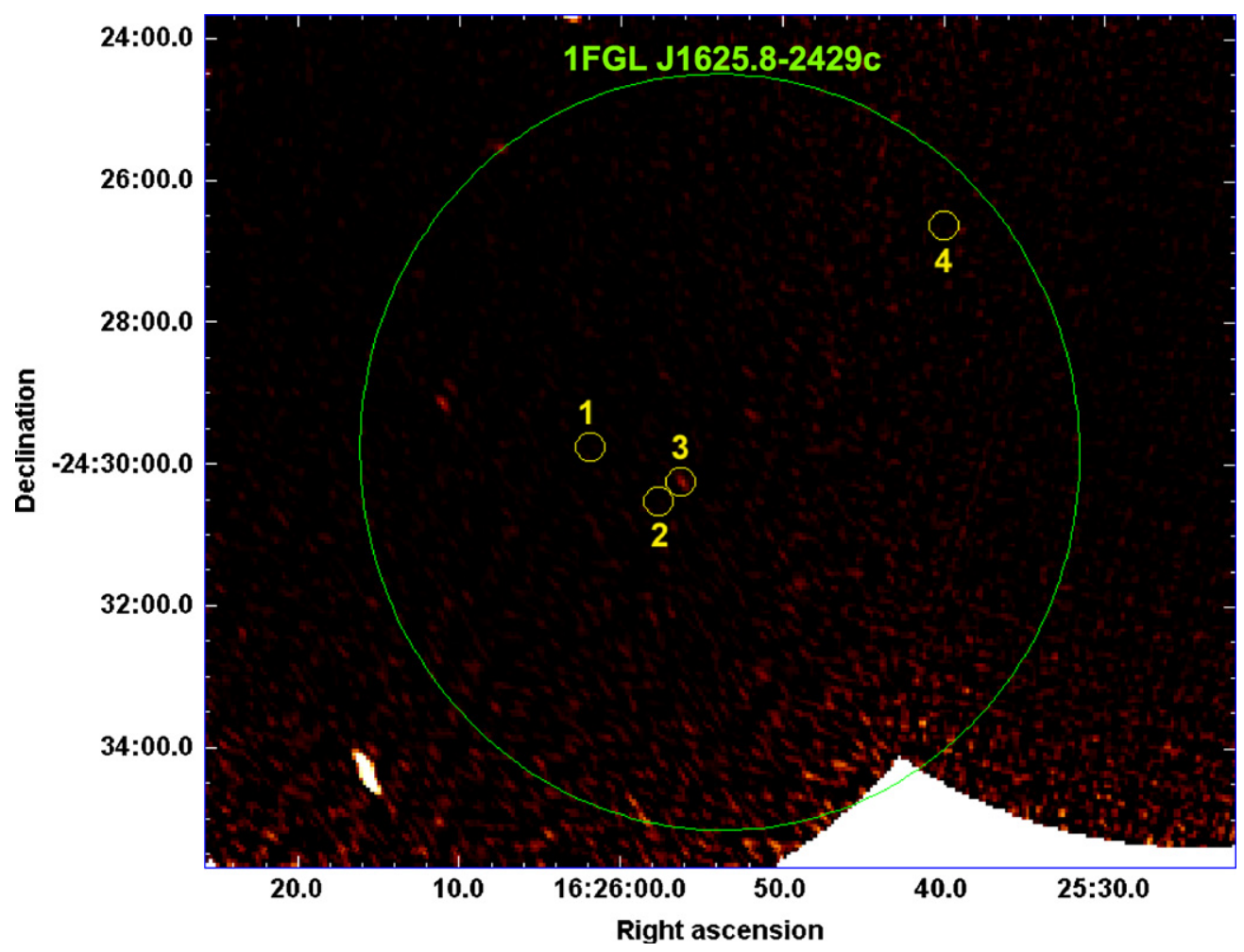

Figure 9. Radio mosaic of the 1FGL J1625.8-2429c error circle as observed with the VLA at the $6 \mathrm{~cm}$ wavelength. Numbers indicate the T Tauri stars consistent with this Fermi source in decreasing order of right ascension. Only the T Tauri star no. 3 is detected in the radio.

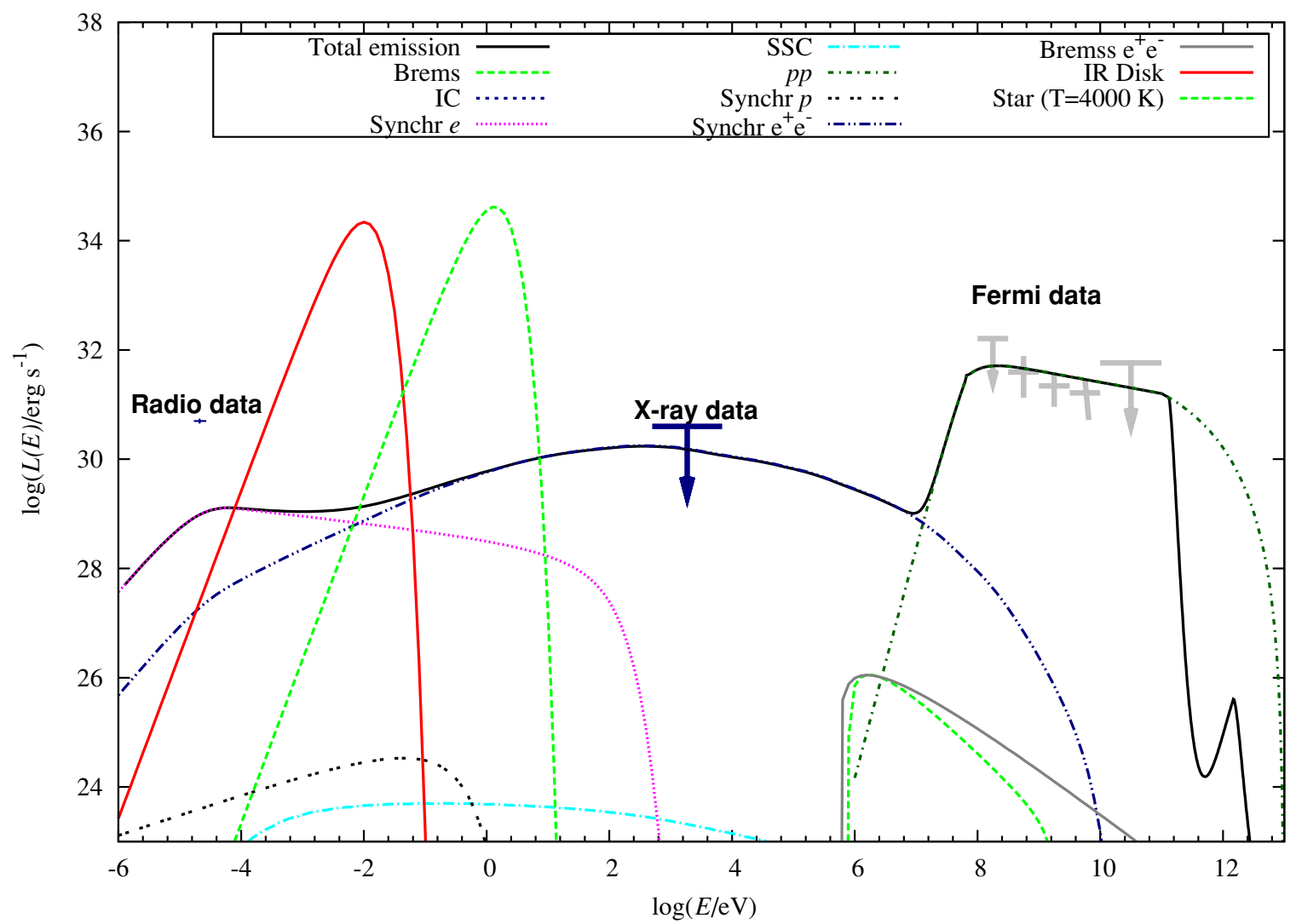

Figure 10. Computed non-thermal luminosity and Fermi upper bounds for the four T Tauri stars, assuming a distance of 120 pc. The spectral energy distribution is corrected by photon absorption. Model parameters as in Table 1 . 
acceleration). The normalization constant is obtained from the energy density of cosmic rays in the solar neighborhood, i.e., $\omega_{\mathrm{CR}} \sim 1 \mathrm{eV} \mathrm{cm}^{-3}$ (e.g., Ginzburg \& Syrovatskii 1964). With these figures and the Fermi flux, we obtain an average density of $\langle n\rangle \sim 10^{4} \mathrm{~cm}^{-3}$, one order of magnitude greater than current estimates $^{7}$ (e.g., Crutcher 1991).

If the effects of the galactic cosmic rays with the interstellar material are responsible for the Fermi source, then 1FGL J1625.8-2429c would be the nearest passive $\gamma$-ray source detected outside the solar system.

\section{CONCLUSIONS}

We have found that under some assumptions $\mathrm{T}$ Tauri stars might be responsible for some nearby Fermi sources. We have presented a simplified model for the high-energy emission of this type of star that agrees with the available multiwavelength observations. T Tauri stars might be a new class of galactic $\gamma$-ray sources in the Galactic plane. Based on this new scenario, 1FGL J1625.8-2429c is the first candidate for collective $\gamma$-ray emission from low-mass protostars. If the association with the $\rho$ Ophiuchi cloud is confirmed, it would be the closest $\gamma$-ray source to the solar system. This statement still holds even in the alternative case where the detected $\gamma$-rays were simply due to cosmic rays interacting with the cloud ambient gas.

More complex models can be developed in the future, using new and deeper observations. The energy where the cutoff lays might be established in the future by new Cherenkov instruments like Cherenkov Telescope Array.

We thank the referee Elisabete M. de Gouveia Dal Pino for constructive and insightful suggestions.

The authors acknowledge support by grant AYA2010-21782C03-03 from the Spanish government, and FEDER funds. This work has been also supported by the Consejería de Innovación, Ciencia y Empresa (CICE) of Junta de Andalucía as research group FQM-322 and excellence fund FQM-5418. This research has made use of the SIMBAD database, operated at CDS, Strasbourg, France. The NRAO is a facility of the NSF operated under cooperative agreement by Associated Universities, Inc. This research also has made use of data from Chandra X-ray Observatory Center, which is operated by the Smithsonian Astrophysical Observatory for and on behalf of the National Aeronautics Space Administration, and of the SpitzerGLIMPSE database, which is operated by the Jet Propulsion Laboratory, California Institute of Technology. G.E.R. and M.V.d.V. are supported by the Argentine Agency CONICET (PIP 0078) and ANPCyT (PICT 2007-00848). G.E.R. also acknowledges Spanish MICINN under grant AYA2010-21782C02-01.

\section{REFERENCES}

Abdo, A. A., Ackermann, M., Ajello, M., et al. (Fermi LAT Collaboration) 2010, ApJS, 188, 405

Aharonian, F. A., \& Atoyan, A. M. 1996, A\&A, 309, 917

Araudo, A. T., Romero, G. E., Bosch-Ramon, V., \& Paredes, J. M. 2007, A\&A, 476, 1289

Aschwanden, M. J. 2008, in IAU Symp. 247, Waves and Oscillations in the Solar Atmosphere: Heating and Magneto-Seismology, ed. R. Erdélyi \& César A. Mendoza-Briceño (Cambridge: Cambridge Univ. Press), 257

Beckwith, S. V. W., Sargent, A. I., Chini, R. S., et al. 1990, AJ, 99, 924

Birk, G. T., Schwab, D., Wiechen, H., et al. 2000, A\&A, 358, 1027

Bosch-Ramon, V., Romero, G. E., Araudo, A. T., \& Paredes, J. M. 2010, A\&A, 511, A8

Casanova, S., Montmerle, T., Feigelson, E. D., et al. 1995, ApJ, 439, 752

Crutcher, R. M. 1991, ApJ, 520, 706

de Gouveia Dal Pino, E. M., \& Lazarian, A. 2005, A\&A, 441, 845

de Gouveia Dal Pino, E. M., Piovezan, P. P., \& Kadowaki, L. H. S. 2010, A\&A, 518, A5

Di Francesco, J., Johnstone, D., Kirk, H., et al. 2008, ApJS, 175, 277

Drury, L. O. 1983, Rep. Prog. Phys., 46, 973

Dutrey, A., Guilloteau, S., \& Simon, M. 1994, A\&A, 286, 149

Feigelson, E. D., \& Montmerle, T. 1999, ARA\&A, 37, 363

Ginzburg, V. L., \& Syrovatskii, S. I. 1964, The Origin of Cosmic Rays (Oxford: Pergamon)

Gomez de Castro, A. I., \& Lamzin, S. A. 1999, MNRAS, 304, L41

Gould, R. J., \& Schréreder, G. P. 1967, Phys. Rev., 155, 1404

Grasdalen, G. L., Strom, K. M., \& Strom, S. E. 1973, ApJ, 184, L53

Günther, H. M., Schmitt, J. H. M. M., Robrade, J., et al. 2007, A\&A, 466, 111

Hayashi, M. R., Shibata, K., \& Matsumoto, R. 1996, ApJ, 468, L37

Johns-Krull, Ch. M., Valenti, J. A., \& Linsky, J. L. 2000, ApJ, 539, 815

Kowal, G., de Gouveia Dal Pino, E. M., \& Lazarian, A. 2011, ApJ, 735, 102

Kowal, G., Lazarian, A., Vishniac, E. T., \& Otmianowska-Mazur, K. 2009, ApJ, 700,63

Lazarian, A., \& Vishniac, E. T. 1999, ApJ, 517, 700

Lin, R. P. 2008, in AIP Conf. Proc. 1039, Particle Acceleration and Transport in the Heliosphere and Beyond: 7th Annual International Astrophysics Conference, ed. G. Li, Q. Hu, O. Verkhoglyadova, G. P. Zank, R. P. Lin, \& J. Luhmann (Melville, NY: AIP), 52

Loinard, L., Torres, R. M., Mioduszewski, A. J., et al. 2008, ApJ, 675, L29

Montmerle, T., \& André, P. 1989, in ESO Conf. Proc. 33, Low Mass Star Formation and Pre-Main Sequence Objects, ed. B. Reipurth (Noordwijk: ESO), 407

Montmerle, T., Koch-Miramond, L., Falgarone, E., et al. 1983, ApJ, 269, 182

Munar-Adrover, P., Paredes, J. P., \& Romero, G. E. 2011, in IAU Symp. 275, Jets at all Scales, ed. G. E. Romero, R. Sunyaev, \& T. Belloni (Cambridge: Cambridge Univ. Press), 406

Muzerolle, J., Calvet, N., Hartmann, L., \& D’Alessio, P. 2003, ApJ, 597, L149

Orellana, M., Bordas, P., Bosch-Ramon, V., et al. 2007, A\&A, 476, 9

Orlando, S., Sacco, G. G., Argiroffi, C., et al. 2010, A\&A, 510, A71

Owocki, S. P., \& Cohen, D. H. 2006, ApJ, 648, 5650

Priest, E., \& Forbes, T. 2000, Magnetic Reconnection: MHD Theory and Applications (Cambridge: Cambridge Univ. Press)

Romero, G. E., Benaglia, P., \& Torres, D. F. 1999, A\&A, 348, 868

Schopper, R., Lesch, H., \& Birk, G. T. 1998, A\&A, 335, 26

Tsuboi, Y., Koyama, K., Murakami, H., et al. 1998, ApJ, 503, 894

Tsuneta, S., \& Naito, T. 1998, ApJ, 495, L67

Vila, G. S. 2010, Int. J. Mod. Phys. D, 19, 659

Vila, G. S., \& Aharonian, F. A. 2009, in Compact Objects and their Emission, ed. G. E. Romero \& P. Benaglia (La Plata: Paideia), 1

Walter, F. M., \& Kuhi, L. V. 1984, ApJ, 284, 194

Wilking, B. A., Lada, C. J., \& Young, E. T. 1989, ApJ, 340, 823

Zenitani, S., \& Hoshino, M. 2001, ApJ, 562, L63

\footnotetext{
7 We remark that the cosmic-ray energy density in $\rho$ Ophiuchi is not known with any accuracy, a fact that has impact in the estimates. However, a difference of one order of magnitude would be, at least, peculiar.
} 